Original research article

\title{
Astragalus polysaccharide alleviates alveolar bone destruction by regulating local osteoclastogenesis during periodontitis
}

\author{
Yakun Han *, Chengcheng Yu, Yan Yu \\ Affiliated Hospital of Jilin Medical University, Department of Stomatology, Jilin, China
}

\begin{abstract}
Inflammatory imbalance of bone formation/resorption leads to alveolar bone destruction. Astragalus polysaccharide has been confirmed to have anti-inflammatory effects. We sought to disclose the protective effect and its potential mechanisms of astragalus polysaccharide in the periodontitis model. Experimental periodontitis was induced by cotton ligatures for this study. We measured the alveolar bone damage rate, periodontal osteoclasts, proportion of CD4+Foxp3+, CD4+IL-10+, CD4+TGF- $\beta+$ subsets in the gingiva, and RANKL, OPG, TGF- $\beta+$, and IL-10+ level in the gingiva. We also cultured osteoclast precursor cells in the presence of RANKL and astragalus polysaccharide. Osteoclasto-like cells were identified by TRAP staining, mRNA of RANK, TRAP, and TRAF6 were evaluated by real time PCR. We found that astragalus polysaccharide caused significant protection of the alveolar bone via reducing local osteoclasts. It also decreased the proportion of CD4+Foxp3+ cells and upregulated the level of CD4+IL-10+ cells, reduced RANKL, and remedied IL-10 levels. In cell culture experiments, astragalus polysaccharide prohibited the RANKL mediated osteoclast differentiation. The findings of this study disclose the functions and possible mechanisms of astragalus polysaccharide engaged in local osteoclastogenesis, and reveal the considerable effect of astragalus polysaccharide in alveolar bone homeostasis and its likely contribution to host immuno-regulation in periodontitis.
\end{abstract}

Keywords: Astragalus polysaccharide; Osteoclastogenesis; Periodontitis; RANKL; Tregs

\section{Highlights:}

- Astragalus polysacharin alleviated periodontal inflammation and protect alveolar bone from damage.

- Astragalus polysacharin decrease Foxp3+ Tregs but increase IL-10+ Tregs.

- Astragalus polysacharin prohibited RANKL, mediated osteoclastogenesis.

\section{Introduction}

As one of the most common oral conditions, periodontitis has been defined as a disease that results from periodontopathogen infection and is distinguished by periodontal inflammation and alveolar bone erosion (Bouziane et al., 2020). An earlier study had shown that periodontal tissue damage is mainly caused by inflammatory responses (Pan et al., 2019). Despite an incompletely understood mechanism, host immunity is considered to be crucial in defense against periodontitis. When the periodontopathogen invades local lesions, the host immune system is activated. Various types of immune cells, inflammatory cytokines, and other elements participate in this process, which leads to effective eradication of the pathogenic bacteria.

Periodontitis often begins with subgingival dental plaque dysbiosis. However, simple dysbiosis is not sufficient to result in severe alveolar bone destruction (Lamont and Hajishen- gallis, 2015). Recently, many immune cells and inflammatory cytokines were found to be involved in the development of periodontitis (Hajishengallis, 2014; Wang and McCauley, 2016). Adaptive immune responses of the host to periodontal pathogens are considered to be the major factors involved with alveolar bone destruction (Van Dyke and van Winkelhoff, 2013). Given the homology of bone cells to immune cells, cells involved in adaptive immune responses have the potential to regulate alveolar bone homeostasis by modulating the production of osteoblasts/osteoclasts and related cytokines (Chen et al., 2016; Hu et al., 2017; Könnecke et al., 2014). This internal relationship between bone and immune systems is called "osteoimmunity” (Ginaldi and De Martinis, 2016). Osteoimmunity is not only responsible for the imbalance of alveolar bone homeostasis in periodontitis but it is also the most effective system to regulate periodontitis.

An imbalance of receptor activator of nuclear factor-kappa B ligand (RANKL) and its competitive inhibitor, osteoprotegerin (OPG), has also been considered to be a crucial cause of

\footnotetext{
* Corresponding author: Yakun Han, Affiliated Hospital of Jilin Medical University, Department of Stomatology, Huashan St. No. 81, Jilin, 132013, China; e-mail: hanyk1986@qq.com

http://doi.org/10.32725/jab.2021.010

Submitted: 2020-07-25 • Accepted: 2021-02-23 • Prepublished online: 2021-04-15

J Appl Biomed 19/2: 97-104 • EISSN 1214-0287 • ISSN 1214-021X

(c) 2021 The Authors. Published by University of South Bohemia in České Budějovice, Faculty of Health and Social Sciences.

This is an open access article under the CC BY-NC-ND license.
} 
periodontal tissue damage, especially the disruption of alveolar bone homeostasis (Iviglia et al., 2019). The RANKL/OPG system has been shown to play a primary role in regulating osteoclast differentiation (López Roldán et al., 2020). The receptor, nuclear factor kappa B receptor activating factor (RANK), is located on the precursor cell membrane surface. When RANKL binds to RANK, multiple signaling cascades are switched on in osteoclast precursor cells to promote their differentiation into osteoclasts. Conversely, when OPG binds to RANK, this process cannot begin. Under physiologic conditions, the RANKL/ OPG ratio in periodontal tissue is maintained within a normal range that not only ensures normal reconstruction and renewal of alveolar bone but also avoids over resorption from abnormal osteoclast activation. During periodontitis, increased RANKL levels in local lesions have been shown to induce over differentiation of osteoclasts, subsequently leading to damage of alveolar bone (Teodorescu et al., 2019).

Hangqi is a traditional Chinese medicine that has been proven to be a remedy for most common diseases (Elabd et al., 2020), such as diabetes, cardiovascular and cerebrovascular diseases, tumor, and autoimmune diseases. Recently, its active constituent, astragalus polysaccharide, was established to alleviate bone destruction in animals with osteoporosis. Besides, researchers proved that astragalus polysaccharide administration protects bone from metabolic damage resulting from oestrogen levels decrease in animals that experienced ovariectomy (Ou et al., 2019), which furtherly indicated a protective effect of astragalus polysaccharide on the host bone system.

Considering these findings, we hypothesized that astragalus polysaccharides could remedy alveolar bone destruction during periodontitis. In this study, we aimed to determine the effect and mechanism of astragalus polysaccharides in keeping host alveolar bone homeostasis by affecting RANKL mediated osteoclastogenesis during periodontitis. Given the important function of Tregs cells in regulation of local osteoclastogenesis, their expression was also identified.

\section{Materials and methods}

\begin{abstract}
Animals
Sprague Dawley rats (Specific Pathogen Free, 200-250 g) were chosen for the present research. All 30 animals were equally and randomly divided into a healthy group (HE, $n=10$ ), experimental periodontitis group (EP, $n=10$ ), and astragalus polysaccharide treatment group (AT, $n=10$ ). Animals from all three groups were fed under the same standard laboratory conditions (humidity, $55 \pm 5 \%$; light period, 8:00-20:00; temperature, $20 \pm 2{ }^{\circ} \mathrm{C}$ ). The protocols for the research procedures and animal surgeries were approved by the Ethics Committee.
\end{abstract}

\section{Periodontitis models}

We induced experimental periodontitis in animals from the EP and AT groups. Experimental periodontitis was induced by cotton ligatures, as shown in earlier studies (Elabd et al., 2020). All rats in the three groups were sacrificed 4 weeks later. Maxillae, gingiva, and gingival crevicular fluid were obtained for the following analyses.

\section{Astragalus polysaccharide administration}

Astragalus polysaccharide ( $500 \mathrm{mg} / \mathrm{kg}$ body weight) was orally gavaged into the esophagus of animals in the treatment group. Drugs were administered $1 \mathrm{~h}$ before the induction of periodontitis and then given daily per the protocol until day 28 .
Animals in the other two groups received the same volume of phosphate buffer saline until they were sacrificed.

\section{Alveolar bone resorption evaluation}

Alveolar bone resorption evaluation was performed as described in other reports (Shi et al., 2020). The resected maxilla samples were fixed with paraformaldehyde (4\%). After $24 \mathrm{~h}$, the samples were transferred into $\mathrm{H}_{2} \mathrm{O}_{2}$ (3\%) overnight. Subsequently, all samples were strained using aqueous methylene blue (1\%) to highlight the differentiation between alveolar bone and teeth. To determine the grade of alveolar bone resorption, alveolar crest reductions at the center of the first molar were measured, and the entire cemento-enamel junction $(C E J)$ to the alveolar bone crest $(A B C)$ was analyzed. We recorded both buccal and palatal CEJ-ABC distances of the target molar. CEJ-ABC distances in mesial, middle, and distal sit of each surfaces were established. The average value of these six predetermined sites was calculated, subsequently was compared among the three groups. All detections were performed three times by two blinded evaluators.

\section{Flow cytometry}

To determine how astragalus polysaccharide affects Tregs differentiation during periodontitis, we determined proportions of these cells in local lesions using flow cytometry. Gums were cut into $1 \mathrm{~mm}^{3}$ sizes and then digested with collagenase type 2 (10 mg/ml), hyaluronidase $(5 \mathrm{mg} / \mathrm{ml})$ and DNase I (0.05\%) for $1 \mathrm{~h}$ at $37^{\circ} \mathrm{C}$ (Ku et al., 2018). Thereafter, the tissue was further disposed by $200 \mu \mathrm{m}$ stainless steel screens. Lymphocytes were purified by Ficoll-Hypaque density-gradient centrifugation. For further detection, lymphocytes were stained with different antibodies groups (CD4 and Foxp3, CD4 and IL-10, CD4 and TGF- $\beta$, Thermo Fisher Scientific). The staining was performed on ice, $30 \mathrm{~min}$, at $4{ }^{\circ} \mathrm{C}$ in darkness according to manufacturer's protocol. Cell analysis was performed with a fluorescence-activated cell sorting (FACS) Calibur flow cytometer. Lymphocytes were gated from the forward and side scattergrams. Data were analyzed with FlowJo software.

\section{ELISA}

We then measured the concentration of some inflammatory cytokines in gingival crevicular fluid with an enzyme-linked immunosorbent assay (ELISA) kit to illustrate how astragalus polysaccharide could affect host immunity during periodontitis, the test was performed as reports (Matsuda et al., 2016). RANKL, OPG, TGF- $\beta$, and IL-10 were detected. GCF obtainment and ELISA tests were performed according to an earlier report.

\section{Real-time PCR}

Gingival samples from each animal were homogenized for RNA extraction. Total RNA was isolated with an RNAiso reagent and then was reverse transcribed to synthesize complementary DNA (cDNA). We subsequently performed real-time polymerase chain reaction (qPCR) in an ABI PRISM cycler with a SYBR Green PCR Kit and specific primers for RANKL, OPG, TGF- $\beta$, IL-10, and $\beta$-actin. Expression values were calculated using the $2-\Delta \Delta C$ t method for every target gene, according to the housekeeping gene. The difference in mRNA expression of the above cytokines was then analyzed. The values of the healthy group were used as a reference (fold change $=1$ ). All experiments were performed independently and in triplicate. Primer sequences are shown in Table 1. 
Table 1. Primer sequences

\begin{tabular}{lll}
\hline Gene & & Sequences \\
\hline$\beta$-actin & -F- & GGTCATCACTATTGGCAACG \\
& -R- & TCCATACCCAAGAAGGAAGG \\
RANKL & -F- & ACCAGCATCAAAATCCCAAG \\
& -R- & TTTGAAAGCCCCAAAGTACG \\
OPG & -F- & CGTCACCCACAGTCTGAGGAA \\
& - R- & TCAACTGCCATTTCAAGAGCC \\
TGF- $\beta$ & -F- & GTGTGGAGCAACATGTGGAACTCTA \\
& - R- & CGCTGAATCGAAAGCCCTGTA \\
IL-10 & - F- & GCCAGAGCCACATGCTCCTA \\
& -R- & GATAAGGCTTGGCAACCCAAGTAA \\
\hline
\end{tabular}

\section{Cell culture and identification}

To determine whether astragalus polysaccharide affect RANKL-mediated osteoclastogenesis, we cultured osteoclast precursors (bone marrow mononuclear cells) in the presence of M-CSF (25 ng/ml) and RANKL (100 ng/ml) in flat-bottomed 96-well plates, as previously described (Huo and Sun, 2016). In the blocking experiment, astragalus polysaccharide $(50 \mathrm{ng} / \mathrm{ml}, 100 \mathrm{ng} / \mathrm{ml}$ ) was added to the culture medium. After a 7-day culture, cells were collected and stained by TRAP kits. TRAP-positive cells containing $\geq 3$ nuclei were considered osteoclast-like cells. The number of osteoclast-like cells in each well was counted and the data was analyzed. Additionally, the mRNA of TARP, RANK, and TNF receptor-associated factor 6 (TRAF6) was quantified to furtherly illustrate the mechanism of how astragalus polysaccharide affects RANKL-mediated osteoclastogenesis. $\beta$-actin (Applied Biosystems, Waltham, MA, USA) was chosen as the housekeeping gene. mRNA detection was performed as described in the previous method (real-time $\mathrm{PCR})$. The results of the normal culture group were used as a benchmark (expression fold $=1$ ). Primer sequences are displayed in Table 1.

\section{Statistical analysis}

All experiments were performed independently and in triplicate. The data are presented as the mean \pm standard error of the mean (SEM). One-way analysis of variance followed by the Bonferroni test or Student's $t$-test was used to compare the means, and the Dunn test was used to compare the medians. A value of $P<0.05$ denoted a statistically significant difference.

\section{Results}

\section{Astragalus polysaccharide affected alveolar bone loss and osteoclast differentiation}

To establish whether astragalus polysaccharide could prevent periodontopathogen-induced alveolar bone loss, we first recorded the distance between $\mathrm{CEJ}$ and $\mathrm{ABC}$ in the first molar and then calculated the resorption ratio of each alveolar bone sample. As shown in Fig. 1, animals in both the experimental periodontitis group and the astragalus polysaccharide treatment group showed higher fold bone resorption than animals in the healthy group $(P<0.05)$. However, we found that astragalus polysaccharide treatment significantly alleviated alveolar resorption as compared with the animals in the experimental periodontitis group (Fig. 1 , mean \pm SEM, each $n=10$ ). These results proved that astragalus polysaccharide treatment ameliorated alveolar bone resorption.

\section{Analysis of Tregs}

To determine the effect of astragalus polysaccharide involvement in local immunoregulation, we assessed the proportion of Foxp3+, IL-10+, and TGF- $\beta+$ subsets in CD4+ cells to establish the level of Treg subpopulations. The results are shown in Fig. 2 (mean \pm SEM, each $n=10)$.

Animals from the EP and AT groups showed a decreased number of Foxp3+, IL-10+, and TGF- $\beta$ + cells compared with those in the HE group $(P<0.05)$. When compared with the two groups, an interesting result was revealed. Despite having similar levels of TGF- $\beta+$ cells $(P>0.05)$, animals from the AT group showed a decrease in Foxp3+ cells and an increase in IL-10+ cells compared with those in the EP group $(P<0.05)$. The results showed that astragalus polysaccharide inhibited the differentiation of Foxp3+ Tregs. However, it seems that this inhibition did not cause an obvious decrease in the function of all Type 1 regulatory ( $\operatorname{Tr} 1)$ cells.

\section{Expression of osteoimmuno-cytokines in local lesions}

Since astragalus polysaccharide treatment significantly alleviated host alveolar resorption, we hypothesized that astragalus polysaccharide modulates cytokine expression, which is closely related to bone homeostasis. To test this hypothesis, we separated the gingiva of each animal and then determined mRNA expression of the cytokines listed above with real-time PCR.
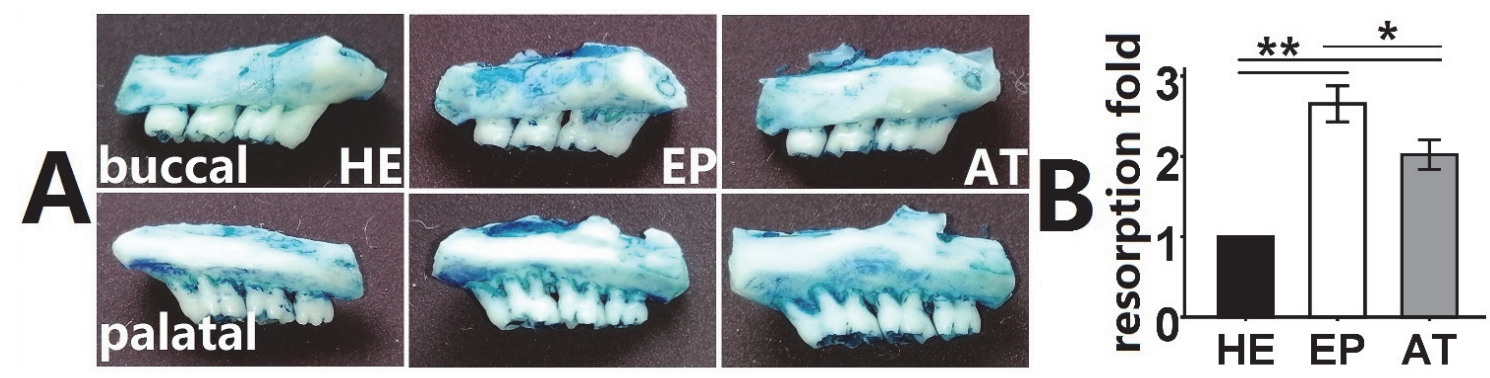

Fig. 1. EP and AT group displayed serious alveolar bone resorption in comparison to the HE group. However, CEJ-ABC distances in $A T$ group showed to be obviously decreased as compared to EP group. (A) Alveolar bone resorption of each group: Image. (B) Alveolar resorption fold of each group $\left(n=10 ;{ }^{*} P<0.05 ;{ }^{* *} P<0.01\right)$. HE - control group; EP - experimental periodontitis group; AT - astragalus polysaccharide treatment group. 


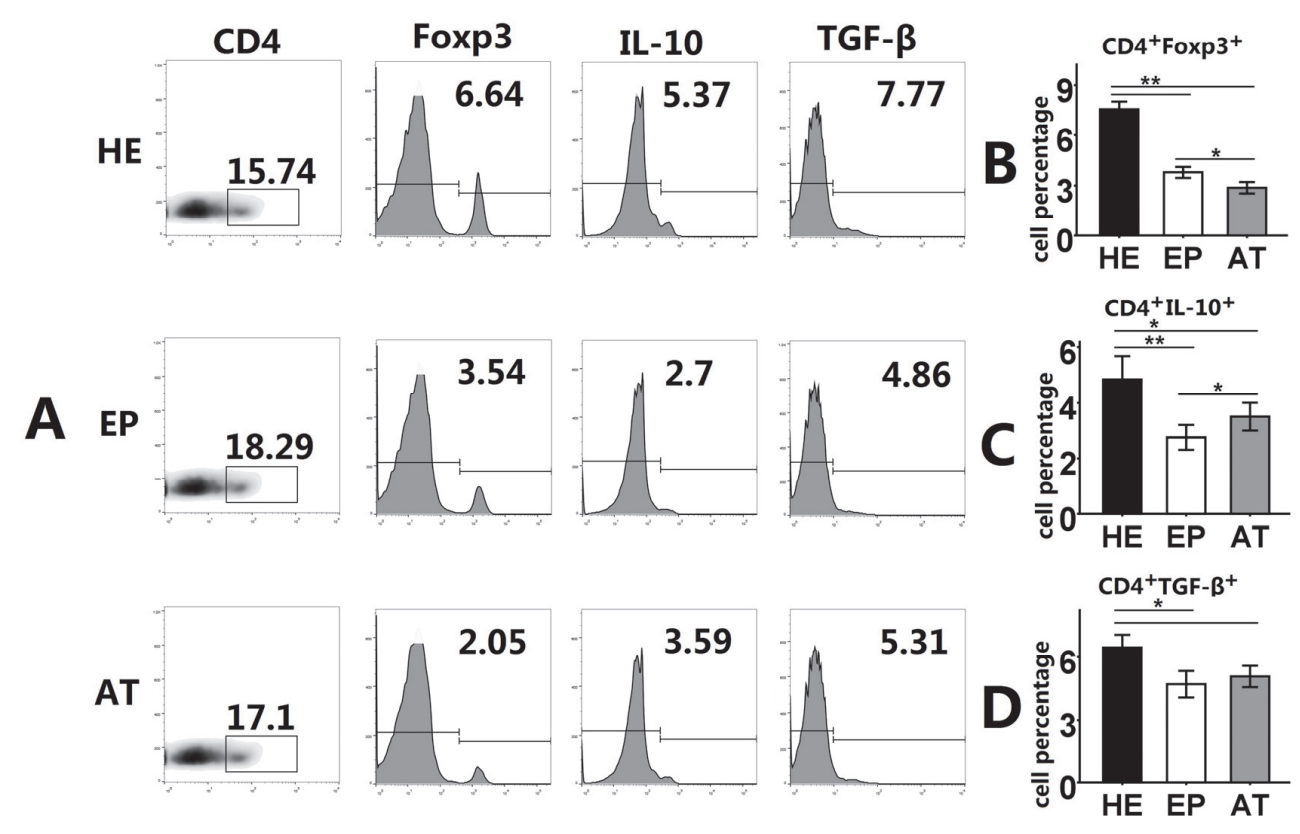

Fig. 2. To determine the effect of astragalus polysaccharide involvement in local Treg regulation, we detected the proportion of Foxp3+ subsets, IL-10+ subsets, and TGF- $\beta$ + subsets in CD4+ cells. Animals from EP and AT group showed a lower level of Foxp3+ subsets, IL-10+ subsets, and TGF- $\beta+$ subsets than the HE group $(P<0.05)$. Despite the similar level of TGF- $\beta+$ subsets $(P>0.05)$, animals from AT group show a lower level of Foxp3+ subsets and a higher level of IL-10+ subsets than the EP group $(P<0.05)$. (A) Percentages of CD4, ror- $\gamma \mathrm{t}$, Foxp3, IL-10 and TGF- $\beta$ positive cells in gingiva: Density plot and Histogram. (B) Percentages of CD4+Foxp3+ cells in gingiva: Statistical analysis $\left(n=10 ;{ }^{*} P<0.05\right.$; $\left.{ }^{* *} P<0.01\right)$. (C) Percentages of CD 4+IL-10+ cells in gingiva: Statistical analysis $\left(n=10 ;{ }^{*} P<0.05\right.$; $\left.{ }^{* *} P<0.01\right)$. (D) Percentages of CD4+TGF- $\beta+$ cells in gingiva: Statistical analysis $\left(n=10 ;{ }^{*} P<0.05 ;{ }^{* *} P<0.01\right)$. HE - control group; EP - experimental periodontitis group; AT - astragalus polysaccharide treatment group.

We first established RANKL and OPG mRNA expression levels. We found significantly higher gingival RANKL expression in animals with periodontitis compared with expression in healthy animals $(P<0.05)$. Moreover, the AT group had markedly decreased RANKL expression compared with that of the EP group $(P<0.05)$. Different from RANKL expression, decreased gingival OPG mRNA expression was only seen in the healthy group compared with the other groups, and no significant differences were seen among the remaining groups $(P>0.05)$ (Fig. 3A, B, mean \pm SEM, each $n=10)$.

Additionally, we determined the IL-10 and TGF- $\beta$ mRNA expression of each group. Compared with the EP group, the HE group expressed higher cytokine levels $(P<0.05)$. There was no significant difference in IL-10 and TGF- $\beta$ expression between the EP and AT groups. Interestingly, although the AT group expressed lower levels of TGF- $\beta$ compared with expression in the HE group $(P<0.05)$, we did not find the same trend in the case of IL-10 (Fig. 3C, D, mean \pm SEM, each $n=10$ ), which indicated a substantial effect of astragalus polysaccharide on the expression of some anti-inflammatory cytokines.

For a more in-depth analysis, we also measured the concentration of the above cytokines in the gingival crevicular fluid. Concentrations of all the above cytokines in each sample were detected using ELISA.

Similar to what was seen at the level of mRNA, RANKL concentrations in the gingival crevicular fluid of animals in the EP group were higher than those in the HE group $(P<0.05)$. Besides, animals in the AT group had lower RANKL concentrations compared with those in the EP group $(P<0.05)$. There were no significant differences in OPG concentrations between animals in the HE group and those in the EP group (Fig. 3E, F, mean $\pm \operatorname{SEM}$, each $n=10$ ).
When we looked at the concentrations of other cytokines, an interesting fact emerged. First, we established that animals in the EP groups had decreased IL-10 and TGF- $\beta$ concentrations compared with the HE group $(P<0.05)$ (Fig. $3 G, H$, mean \pm SEM, each $n=10)$, which was similar to that seen in the gingiva. Despite a little increase in the concentration of cytokines from the AT group, no clear differences were appreciated between EP and AT groups. However, when compare the $\mathrm{HE}$ and AT group, we find that IL-10 and TGF- $\beta$ concentrations did not differ among animals in either of the two groups $(P>0.05)$ (Fig. 3G, H, mean \pm SEM, each $n=10)$. Together, these data suggest that astragalus polysaccharide is predominately involved in local bone homeostasis by regulating some inflammatory cytokines which are closely related to osteoimmuno-response.

\section{Astragalus polysaccharide prohibited RANKL mediated osteoclasts differentiation}

The above outcome determined that astragalus polysaccharide decreased RANKL expression in local lesions, which indicated an indirect prohibition of osteoclastogenesis. Given the function of RANKL on osteoclast differentiation, we also hypothesized that astragalus polysaccharide may have a direct effect on osteoclastogenesis. To confirm this, we cultured bone marrow mononuclear cells in presence of RANKL and astragalus polysaccharide in vitro. We counted the number of osteoclasts and then investigated the level of mRNA of TARP, RANK, and TRAF6 genes closely related to RANKL mediated osteoclast differentiation.

RANKL induced significantly higher osteoclast-like cell formation (Fig. $4 \mathrm{~A}, \mathrm{~B}, P<0.05$, mean \pm SEM, each $n=10$ ) when 

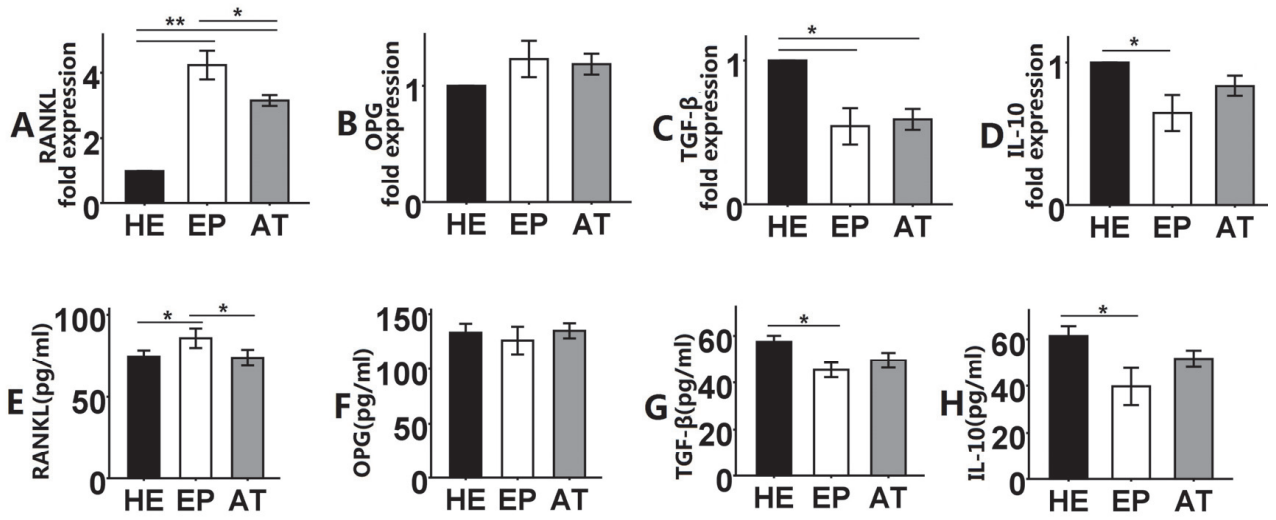

Fig. 3. To evaluate how astragalus polysaccharide affects local osteoclastogenesis, we determined the level of RANKL, OPG, IL-10, and TGF- $\beta$ in local lesions by real time PCR and ELISA. In gingiva, the RANKL level which comes from animals with periodontitis showed to be significantly higher than that in healthy animals $(P<0.05)$. AT group markedly expressed less RANKL than the EP group $(P<0.05)$. There was no significant difference between the remaining groups in OPG expression $(P>0.05)$. Compared with individuals from the EP group, the healthy ones expressed higher IL-10 and TGF- $\beta$ levels $(P<0.05)$. However, the HE group did not show obviously higher IL-10 level in comparison with AT group $(P>0.05)$. There was no significant difference between AT group and the EP group in IL-10 and TGF- $\beta$ levels $(P>0.05)$. In GCF, the EP group showed higher RANKL level than the HE and AT group $(P<0.05)$; the HE group showed higher IL-10 and TGF- $\beta$ levels than EP and AT groups $(P<0.05)$; There was no other significant difference in other comparisons $(P>0.05)$. (A) Level of RANKL mRNA in gingiva $(n=10$; $\left.{ }^{*} P<0.05{ }^{* *} P<0.01\right)$. (B) Level of OPG mRNA in gingiva $\left(n=10 ;{ }^{*} P<0.05\right.$; $\left.{ }^{* *} P<0.01\right)$. (C) Level of TGF- $\beta$ mRNA in gingiva $(n=10$; ${ }^{*} P<0.05$; ** $\left.P<0.01\right)$. (D) Level of IL-10 mRNA in gingiva $\left(n=10 ;{ }^{*} P<0.05\right.$; $\left.{ }^{*} P<0.01\right)$. (E) Concentration of RANKL in GCF $(n=10$; ${ }^{*} P<0.05$; $\left.{ }^{*} P<0.01\right)$. (F) Concentration of OPG in GCF $\left(n=10\right.$; $^{*} P<0.05$; $\left.{ }^{* *} P<0.01\right)$. (G) Concentration of TGF- $\beta$ in GCF $\left(n=10\right.$; ${ }^{*} P<0.05$; $\left.{ }^{* *} P<0.01\right)$. (H) Concentration of IL-10 in GCF $\left(n=10 ;{ }^{*} P<0.05 ;{ }^{* *} P<0.01\right)$. HE - control group; EP - experimental periodontitis group; $\mathrm{AT}$ - astragalus polysaccharide treatment group.

the cells were cultivated in RANKL only cultivation medium (without astragalus polysaccharide). In cultures with higher concentration (100 ng/ml) of astragalus polysaccharide $(P<0.05)$, only a small number of osteoclast-like cells were observed, suggesting that astragalus polysaccharide prohibited osteoclast-like cells formation via RANKL manner. More- over, astragalus polysaccharide adequately prohibited osteoclast-like cells formation from bone marrow mononuclear cells as determined by the reduced expression of related genes (Fig. 4C, D, $P<0.05$, mean \pm SEM, each $n=10$ ) we chose, compared with controls.

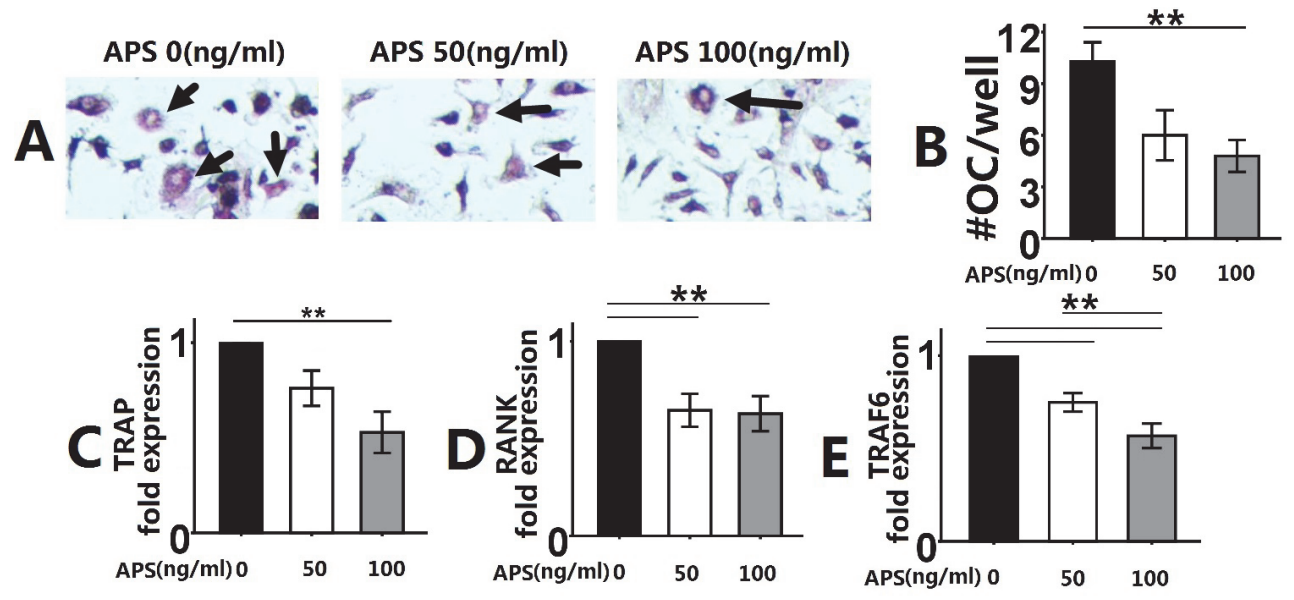

Fig. 4. Bone marrow mononuclear cells (as osteoclasts precursor) were cultured in presence of RANKL (100 ng/ml) independently or with astragalus polysaccharide $(50 \mathrm{ng} / \mathrm{ml}, 100 \mathrm{ng} / \mathrm{ml})$ to determine the potential effect of astragalus polysaccharide on RANKL mediated osteoclastogenesis. The results showed that a higher concentration of astragalus polysaccharide reduces the differentiation of TRAP+ osteoclast-like cells. Meanwhile, astragalus polysaccharide reduced the expression of TRAP, RANK, and TRAF6, which are closely related to the RANKL signal. (A) TRAP positive cells in different culture medium $(100 \times)$. (B) Number of osteoclasts in each culture well $\left(n=10 ;{ }^{*} P<0.05\right.$; $\left.{ }^{* *} P<0.01\right)$. (C) Expression fold of TRAP mRNA in each group $\left(n=10 ;{ }^{*} P<0.05 ;{ }^{* *} P<0.01\right)$. (D) Expression fold of RANK mRNA in each group $\left(n=10 ;{ }^{*} P<0.05\right.$; $\left.^{* *} P<0.01\right)$. (E) Expression fold of TRAF6 mRNA in each group $\left(n=10\right.$; $^{*} P<0.05$; $\left.{ }^{* *} P<0.01\right)$. HE - control group; $\mathrm{EP}$ - experimental periodontitis group; AT - astragalus polysaccharide treatment group. 


\section{Discussion}

As a traditional Chinese medicine, Huangqi has a long history of use. It has been confirmed to be effective in inducing anti-inflammatory, anti-tumor, and immunomodulatory responses even though its structure has not been completely understood (Fu et al., 2014). Astragalus polysaccharide is a key macromolecule in Huangqi. It has been proved that astragalus polysaccharide enhances proliferation and differentiation in either B or T cells, which is involved in lots of diseases (Wu et al., 2018).

Given the immunomodulatory function of Tregs, they were recognized to be a crucial regulator of the periodontitis inflammatory process. Tregs are often identified by CD4 and CD25 expression and they are divided into two subsets: innate Tregs (Foxp3+) and adaptive (Foxp3-) Tregs (Terhune and Deth, 2014). Innate Tregs are responsible for regulating autoimmune responses, while adaptive Tregs often regulate infective immune responses (Ku et al., 2018). Tregs generally suppress the immune response by producing anti-inflammatory cytokines, such as IL-10 and TGF- $\beta$ (Kumar et al., 2018). During periodontitis, Tregs numbers increase proportionally in both local lesions and systemically as inflammation develops. Increased Tregs regulate local inflammation, limit it to a certain extent, which avoids excessive tissue injure resulting from the serious immune response. On the other hand, these increased Tregs produce more cytokines which are proved to be crucial in osteoclastogenesis, thereafter resulting in an imbalance of alveolar bone homeostasis.

In our study, astragalus polysaccharide showed significantly pleiotropic effects in Treg regulation. Astragalus polysaccharide significantly downregulated Foxp3+ Treg levels, which correlates with the results of the former studies (Zheng et al., 2020). Previous reports confirmed that astragalus polysaccharide was engaged in the proliferation and polarization of CD4+T cells, thereafter affecting the T cell-mediated immunity (Xue et al., 2020). This effect could be result from Foxp3 suppression. Similar to the present study, earlier research also described Foxp3 suppression in animals treated by astragalus polysaccharide (Li et al., 2012). Foxp3, the remarkable identification of innate Tregs, dominates their immuno-suppressive function. Co-culturing of CD4+Tregs with astragalus polysaccharide lead to a lower Foxp3 expression. Importantly, these CD4+Tregs could only show a weak immunosuppression (Liu et al., 2011). Although the whole mechanism is still unclear, toll-like receptor 4 (TLR4) might be crucial in this process. It is now generally accepted that TLR4 has a major role in constraining LPS mediated inflammation response (Płóciennikowska et al., 2015). The most convincing evidence comes from in vivo and ex vivo experiments in animals where pathogen infection or LPS injection enhanced TLR4 expression in Tregs. Researchers established that the TLR4 blockade prohibited the effect of astragalus polysaccharide on Tregs, and TLR4 manipulation enhanced the influence (Ren et al., 2018).

In addition to Foxp3 expression, we confirmed that astragalus polysaccharides increased the number of IL-10+ Treg cells but had no obvious effects on TGF- $\beta$ expression, suggesting that astragalus polysaccharide could have the potential to activate Tr1 cells. Correspondingly, IL-10 level in local lesions was remedied by astragalus polysaccharides administration during periodontitis. As a potent anti-inflammatory factor, IL-10 inhibits local inflammation and protects against alveolar bone destruction. IL-10 inhibits osteoclastogenesis by inhibiting RANKL expression and NFATc1 function (Kawamoto et al.,
2016). Moreover, IL-10 promotes osteoblast differentiation by inhibiting TGF- $\beta$ expression (Zhang et al., 2014). When researchers knocked out the IL-10 gene in animals, osteoblast deficiency, and alveolar bone resorption were seen (Claudino et al., 2010). These mechanisms further enhanced the protective effect of astragalus polysaccharides on alveolar bone during periodontitis.

Administration of astragalus polysaccharides not only modulates differentiation and polarization of host $\mathrm{CD} 4+$ Foxp3+ Tregs but also reduces the level of RANKL, which subsequently results in the decrease of local osteoclastogenesis (Wang et al., 2019). In the present study, we found that the administration of astragalus polysaccharide effectively alleviated alveolar bone destruction in animals with periodontitis. Alveolar bone homeostasis in periodontitis depends primarily on osteoblast-mediated bone formation and osteoclast-mediated bone destruction (Prieto Damm et al., 2013). In this process, RANKL-induced osteoclastogenesis plays a crucial role. Animal trials and clinical studies have confirmed the importance of RANKL in periodontal bone resorption (Kuritani et al., 2018). As is reported, RANKL/OPG expression in the gingiva of patients with chronic periodontitis was significantly higher than that of healthy individuals. RANKL levels were also significantly higher in the body fluid samples of the patients, including gingival sulcus fluid, saliva, and peripheral blood (Verde et al., 2015). RANKL concentrations increased gradually from healthy individuals to individuals with gingivitis and then to periodontitis, no matter if measured in local lesions or systemically. In our study, astragalus polysaccharide effectively reduced RANKL concentrations. Astragalus polysaccharide administration promoted a shifting from Th2 to Th1, thereafter down-regulated IL-4 (Zhou et al., 2018). IL-4 plays an essential role in the maturation and function of effector $B$ cells, by co-operating with BAFF (Granato et al., 2014). Generally, periodontal RANKL mainly expressed by B cells. Therefore, reduced IL-4 indirectly results in a decrease of local RANKL level.

Also, we show herein that astragalus polysaccharides not just prohibited RANKL expression, but also modulated RANKL mediated osteoclastogenesis. In cell culture experiments, we demonstrated that elevated astragalus polysaccharides concentration reduced the differentiation of osteoclast-like cells by down-regulating the expression of crucial molecules in a RANK-RANKL signalling, including RANK, TRAP, and TRAF6. As a receptor of RANKL, RANK is located on the surface of the osteoclast precursor cell. When RANK binds RANKL, its intracellular part is activated and then combined with TRAF6 (Walsh and Choi, 2014). This combination promotes phosphorylation of inhibitory $\kappa$ kinases and subsequently activates nuclear factor $\kappa-B$ pathways, which results in the expression of TRAP and maturation of osteoclasts.

Besides osteoclastogenesis, astragalus polysaccharides were shown to promote osteogenesis in a variety of ways. When co-cultured with osteoblasts, astragalus polysaccharides significantly increased alkaline phosphatase and calcium levels in osteoblasts with nodular formations (Gao et al., 2015). Moreover, it has also been demonstrated that astragalus polysaccharide promotes osteoblast differentiation from bone marrow mesenchymal stem cells, which also protects alveolar bone from inflammatory resorption (Jian et al., 2015).

\section{Conclusions}

In conclusion, our study confirmed the pleiotropic immunomodulatory effect of astragalus polysaccharide on Tregs dur- 
ing periodontitis. Of note, the presented research determined, for the first time, that astragalus polysaccharide reduces periodontal osteoclastogenesis by decreasing local RANKL expression and prohibiting RANKL mediated osteoclast differentiation. All our findings highlight the function of astragalus polysaccharide on protection of alveolar bone from inflammatory erosion. What is more, these findings further illustrated the function of astragalus polysaccharide in the remedy of periodontitis. Nevertheless, further investigations are still needed to uncover more detailed mechanisms.

\section{Conflict of interests}

The authors have no conflict of interests to declare.

\section{Acknowledgements}

This work was supported by grants from Science and Technology Plan of Chinese Traditional Medicine (2018127), Province Jilin, China. The authors declare that they have no competing interests related to this study.

\section{References}

Bouziane A, Hamdoun R, Abouqal R, Ennibi O (2020). Global Prevalence of aggressive periodontitis: A systematic review and meta-analysis. J Clin Periodontol 47(4): 406-428. DOI: 10.1111/ jcpe.13266.

Chen Y, Bai P, Liu L, Han J, Zeng H, Sun Y (2016). Increased RANKL expression in peripheral $\mathrm{T}$ cells is associated with decreased bone mineral density in patients with COPD. Int J Mol Med 38(2): 585-593. DOI: 10.3892/ijmm.2016.2629.

Claudino M, Garlet TP, Cardoso CRB, de Assis GF, Taga R, Cunha FQ, et al. (2010). Down-regulation of expression of osteoblast and osteocyte markers in periodontal tissues associated with the spontaneous alveolar bone loss of interleukin-10 knockout mice. Eur J Oral Sci 118(1): 19-28. DOI: 10.1111/j.16000722.2009.00706.x.

Elabd H, Wang H-P, Shaheen A, Matter A (2020). Astragalus membranaceus nanoparticles markedly improve immune and anti-oxidative responses; and protection against Aeromonas veronii in Nile tilapia Oreochromis niloticus. Fish Shellfish Immunol 97: 248-256. DOI: 10.1016/j.fsi.2019.12.025.

Fu J, Wang Z, Huang L, Zheng S, Wang D, Chen S, et al. (2014). Review of the botanical characteristics, phytochemistry, and pharmacology of Astragalus membranaceus (Huangqi). Phytother Res 28(9): 1275-1283. DOI: 10.1002/ptr.5188.

Gao T, Zhang N, Wang Z, Wang Y, Liu Y, Ito Y, Zhang P (2015). Biodegradable Microcarriers of Poly(Lactide-co-Glycolide) and Nano-Hydroxyapatite Decorated with IGF-1 via Polydopamine Coating for Enhancing Cell Proliferation and Osteogenic Differentiation. Macromol Biosci 15(8): 1070-1080. DOI: $10.1002 / \mathrm{mabi} .201500069$.

Ginaldi L, De Martinis M (2016). Osteoimmunology and Beyond. Curr Med Chem 23(33): 3754-3774. DOI: 10.2174/09298673236 66160907162546.

Granato A, Hayashi EA, Baptista BJA, Bellio M, Nobrega A (2014). IL-4 regulates Bim expression and promotes $B$ cell maturation in synergy with BAFF conferring resistance to cell death at negative selection checkpoints. J Immunol 192(12): 5761-5775. DOI: 10.4049/jimmunol.1300749.

Hajishengallis G (2014). Immunomicrobial pathogenesis of periodontitis: keystones, pathobionts, and host response. Trends Immunol 35(1): 3-11. DOI: 10.1016/j.it.2013.09.001.

Hu XL, Sun Y, Xu W, Lin T, Zeng H (2017). Expression of RANKL by peripheral neutrophils and its association with bone mineral density in COPD. Respirology 22(1): 126-132. DOI: 10.1111/ resp.12878.
Huo J, Sun X (2016). Effect of Astragalus polysaccharides on ovariectomy-induced osteoporosis in mice. Genet Mol Res 15(4): 1-9. DOI: 10.4238/gmr15049169.

Iviglia G, Kargozar S, Baino F (2019). Biomaterials, Current Strategies, and Novel Nano-Technological Approaches for Periodontal Regeneration. J Funct Biomater 10(1): 3. DOI: $10.3390 / j f b 10010003$.

Jian J, Sun L, Cheng X, Hu X, Liang J, Chen Y (2015). Calycosin-7-O$\beta$-d-glucopyranoside stimulates osteoblast differentiation through regulating the BMP/WNT signaling pathways. Acta Pharm Sin B 5(5): 454-460. DOI: 10.1016/j.apsb.2015.06.005.

Kawamoto D, Ando-Suguimoto ES, Bueno-Silva B, DiRienzo JM, Mayer MPA (2016). Alteration of Homeostasis in Pre-osteoclasts Induced by Aggregatibacter actinomycetemcomitans CDT. Front Cell Infect Microbiol 6: 33. DOI: 10.3389/fcimb.2016.00033.

Könnecke I, Serra A, Khassawna TE, Schlundt C, Schell H, Hauser A, et al. (2014). T and B cells participate in bone repair by infiltrating the fracture callus in a two-wave fashion. Bone 64: 155-165. DOI: 10.1016/j.bone.2014.03.052.

Ku M, Ke E, Sabouri-Ghomi M, Abadejos JR, Freeman B, Nham A, et al. (2018). Deconstructive somatic cell nuclear transfer reveals novel regulatory T-cell subsets. J Allergy Clin Immunol 142(3): 997-1000. DOI: 10.1016/j.jaci.2018.04.038.

Kumar P, Bhattacharya P, Prabhakar BS (2018). A comprehensive review on the role of co-signaling receptors and Treg homeostasis in autoimmunity and tumor immunity. J Autoimmun 95: 77-99. DOI: 10.1016/j.jaut.2018.08.007.

Kuritani M, Sakai N, Karakawa A, Isawa M, Chatani M, NegishiKoga T, et al. (2018). Anti-mouse RANKL Antibodies Inhibit Alveolar Bone Destruction in Periodontitis Model Mice. Biol Pharm Bull 41(4): 637-643. DOI: 10.1248/bpb.b18-00026.

Lamont RJ, Hajishengallis G (2015). Polymicrobial synergy and dysbiosis in inflammatory disease. Trends Mol Med 21(3): 172-183. DOI: 10.1016/j.molmed.2014.11.004.

Li Q, Bao J-M, Li X-L, Zhang T, Shen X-H (2012). Inhibiting effect of Astragalus polysaccharides on the functions of CD4+CD25 highTreg cells in the tumor microenvironment of human hepatocellular carcinoma. Chin Med J (Engl) 125(5): 786-793.

Liu Q-Y, Yao Y-M, Yu Y, Dong N, Sheng Z-Y (2011). Astragalus polysaccharides attenuate postburn sepsis via inhibiting negative immunoregulation of CD4+CD25(high) T cells. PLoS One 6(6): e19811. DOI: 10.1371/journal.pone.0019811.

López Roldán A, García Giménez JL, Alpiste Illueca F (2020). Impact of periodontal treatment on the RANKL/OPG ratio in crevicular fluid. PLoS One 15(1): e0227757. DOI: 10.1371/journal. pone. 0227757

Matsuda S, Movila A, Suzuki M, Kajiya M, Wisitrasameewong W, Kayal R, et al. (2016). A novel method of sampling gingival crevicular fluid from a mouse model of periodontitis. J Immunol Methods 438: 21-25. DOI: 10.1016/j.jim.2016.08.008.

Ou L, Wie P, Li M, Gao F (2019). Inhibitory effect of Astragalus polysaccharide on osteoporosis in ovariectomized rats by regulating FoxO3a /Wnt signaling pathway. Acta Cir Bras 34(5): e201900502. DOI: 10.1590/s0102-865020190050000002.

Pan W, Wang Q, Chen Q (2019). The cytokine network involved in the host immune response to periodontitis. Int J Oral Sci 11(04): 276-288. DOI: 10.1038/s41368-019-0064-z.

Płóciennikowska A, Hromada-Judycka A, Borzęcka K, Kwiatkowska K (2015). Co-operation of TLR4 and raft proteins in LPS-induced pro-inflammatory signaling. Cell Mol Life Sci 72(3): 557-581. DOI: 10.1007/s00018-014-1762-5.

Prieto Damm D, Sanz Ruiz A, Chaparro Padilla A, Inostroza Silva C, Ramírez Lobos V (2013). Relación entre los niveles de compuestos volátiles sulfurados con los niveles de RANKL y OPG en pacientes con periodontitis crónica moderada o severa: Estudio transversal. Revista Clínica de Periodoncia, Implantología y Rehabilitación Oral 6(1): 20-24. DOI: 10.1016/S0718-5391(13)70115-0.

Ren Q, Zhao S, Ren C, Ma Z (2018). Astragalus polysaccharide alleviates LPS-induced inflammation injury by regulating miR-127 in H9c2 cardiomyoblasts. Int J Immunopathol Pharmacol 32: 2058738418759180. DOI: 10.1177/2058738418759180. 
Shi T, Jin Y, Miao Y, Wang Y, Zhou Y, Lin X (2020). IL-10 secreting B cells regulate periodontal immune response during periodontitis. Odontology 108(3): 350-357. DOI: 10.1007/s10266-019-00470-2.

Teodorescu AC, Martu I, Teslaru S, Kappenberg-Nitescu DC, Goriuc A, Luchian I, et al. (2019). Assessment of Salivary Levels of RANKL and OPG in Aggressive versus Chronic Periodontitis. J Immunol Res 2019. DOI: 10.1155/2019/6195258.

Terhune TD, Deth RC (2014). A role for impaired regulatory T cell function in adverse responses to aluminum adjuvant-containing vaccines in genetically susceptible individuals. Vaccine 32(40): 5149-5155. DOI: 10.1016/j.vaccine.2014.07.052.

Van Dyke TE, van Winkelhoff AJ (2013). Infection and inflammatory mechanisms. J Periodontol 40(Suppl. 14): S1-7. DOI: 10.1111/ jcpe.12088.

Verde ME, Bermejo D, Gruppi A, Grenón M (2015). Effect of Bisphosphonates on the Levels of Rankl and Opg in Gingival Crevicular Fluid of Patients with Periodontal Disease and Post-menopausal Osteoporosis. Acta Odontol Latinoam 28(3): 215-221.

Walsh MC, Choi Y (2014). Biology of the RANKL-RANK-OPG System in Immunity, Bone, and Beyond. Front Immunol 5: 511. DOI: 10.3389/fimmu.2014.00511.

Wang C-WJ, McCauley LK (2016). Osteoporosis and Periodontitis. Curr Osteoporos Rep 14(6): 284-291. DOI: 10.1007/s11914-0160330-3.
Wang W, Liu Q-B, Jing W (2019). Astragalus membranaceus improves therapeutic efficacy of asthmatic children by regulating the balance of Treg/Th17 cell. Chin J Nat Med 17(04): 252-263. DOI: 10.1016/S1875-5364(19)30029-9.

Wu X, Zhou W, Wei Q, Chen P, Li Y (2018). Cytoprotective effects of the medicinal herb Astragalus membranaceus on lipopolysaccharide-exposed cells. Mol Med Rep 18(5): 4321-4327. DOI: $10.3892 / \mathrm{mmr} .2018 .9483$.

Xue L, Wang D, Zhang D, Ju A, Duan A, Xing J, et al. (2020). The immune adjuvant effect of Astragalus polysaccharide on in ovo injection of Newcastle disease vaccine. J Anim Physiol Anim Nutr (Berl) 104(6): 1719-1726. DOI: 10.1111/jpn.13388.

Zhang Q, Chen B, Yan F, Guo J, Zhu X, Ma S, Yang W (2014). Interleukin-10 inhibits bone resorption: a potential therapeutic strategy in periodontitis and other bone loss diseases. Biomed Res Int 2014: 284836. DOI: 10.1155/2014/284836.

Zheng Y, Ren W, Zhang L, Zhang Y, Liu D, Liu Y (2020). A Review of the Pharmacological Action of Astragalus Polysaccharide. Front Pharmacol 11: 349. DOI: 10.3389/fphar.2020.00349.

Zhou X, Liu Z, Long T, Zhou L, Bao Y (2018). Immunomodulatory effects of herbal formula of astragalus polysaccharide (APS) and polysaccharopeptide (PSP) in mice with lung cancer. Int J Biol Macromol 106: 596-601. DOI: 10.1016/j.ijbiomac.2017.08.054. 